\title{
Optimisation of a velocity feedback controller to minimise kinetic energy and maximise power dissipation
}

Michele Zilletti ${ }^{\text {a }}$

Paolo Gardonio $^{\text {a }}$

Stephen J. Elliott ${ }^{\mathrm{b}}$ michele.zilletti@uniud.it

paolo.gardonio@uniud.it

sje@isvr.soton.ac.uk

\footnotetext{
a DIEGM, Università degli Studi di Udine,Via delle Scienze, 208, 33100 Udine, Italy

${ }^{\mathrm{b}}$ Institute of Sound and Vibration Research, Highfield, Southampton SO171BJ, UK
}

Keywords: Active Vibration absorber, Velocity feedback loop, Power absorption, inertial actuator

\begin{abstract}
In this study the active vibration control of a structure modelled as a single degree of freedom system and excited by a white noise force is considered. The control system consists of an inertial actuator driven with a signal proportional to the velocity of the structure under control measured by an ideal collocated sensor. The optimisation of the physical and control parameters of the control system such as the internal damping of the actuator, its natural frequency and the feedback gain of the controller are considered such that either the kinetic energy of the host structure is minimised or the power dissipated by the control system is maximised. This type of control system is only conditionally stable therefore a stability condition has to be satisfied by the optimisation process. The paper shows that the two optimisation criteria are equivalent.
\end{abstract}

\section{INTRODUCTION}

Passive tuned vibration absorbers were invented by Farhm [1] in 1911 and since then have been widely used to control structural vibrations. They consist of a block mass mounted on a elastic suspension, which can usually be modelled as a single degree of freedom system. When they are used to control the vibration of flexible structures subjected to broadband excitation their natural frequency and internal damping are tuned to damp the structural response in a narrow frequency band around a structural resonance [2]. Optimisation criteria 
of passive tuneable vibration absorbers have been proposed by many researchers and a vast literature can be found on this topic [2-12]. A summary of few optimisation criteria can be found in reference [12]. The main limits of these devices is that their efficiency depends on the mass of the device, which implies high added weight to the structure, and is limited to a narrow frequency band.

A substantial improvement can be achieved by using an active vibration absorber, which consists of a passive tuneable vibration absorber with a reactive actuator in parallel with the suspension, which is driven to implement a velocity feedback loop. An example of this device is the voice coil inertial actuator with an accelerometer sensor at its footprint commonly used in vibration control applications [13]. The advantages of an active vibration absorber are its adaptability to parameters changes of the structure under control, which guarantees a better vibration control performance in a wider range of operating conditions compared with a passive one. Depending on the circumstances, active solutions may be cheaper and lighter than passive systems. In some applications active control systems can offer performances that no passive system can achieve, especially in controlling low frequency vibration. A simple way of driving these devices to damp the structural response of flexible structures subjected to broadband excitation is with a velocity feedback loop [14-17]. In this way the control system acts as a sky-hook active damper in the frequency region above the resonance of the actuator over a frequency band of several decades, before higher order resonances interfere with their dynamics $[13,17,18]$. However the $180^{\circ}$ degrees phase shift in the response of the actuator due to its resonance makes the system only conditionally stable also in the ideal case although the stability margin could be improved using a compensator $[17,19]$.

One important issue with such a control system is how the physical properties of the inertial actuator, such as its natural frequency and the internal damping ratio and the gain of the feedback loop are set to optimally control the vibration of the hosting structure [20]. The optimum parameters and the feedback gain are generally a compromise between performance and stability, and the values change depending on the location of the inertial actuator on the structure and the type of primary excitation. In an ideal system, all these parameters would be adjusted to minimise the kinetic energy of the structure under control [15]. However this optimisation may be difficult to implement in practice because it would require velocity measurements in many points of the structure. Previous studies have proposed the power absorbed by the controller as a cost function to be maximised showing that it can be easily 
estimated within the control system [21-24]. For broadband excitation, when stable skyhook active damping is implemented either with a reactive actuator or an inertial actuator, it has been shown both with numerical simulations and experiments that the minimisation of the kinetic energy of the hosting structure and the maximisation of the power absorbed by the control system produce equivalent vibration control effects $[19,25,26]$.

In this paper an inertial actuator that implements an ideal velocity feedback loop is used to control a single mode of a vibrating structure, modelled as a single degree of freedom system, which is subjected to white noise excitation. Two different optimisations are compared: the minimisation of the kinetic energy of the hosting structure and the maximisation of the power absorbed by the control system. It is analytically demonstrated that the two methods are equivalent for the optimisation of the internal damping of the inertial actuator, its natural frequency and feedback gain. Moreover it is demonstrated that the power input into the system only depends on the mass of the primary structure when ideal white noise excitation is assumed. It is also shown that when the optimisation considers only the feedback gain as tuning parameter, analytical expressions for the maximum stable gain and optimum gain can be found by solving quadratic equations.

\section{MODELLING}

Typically when an inertial actuator is used to implement a velocity feedback loop to control the resonant response due to one mode of a flexible structure, a simplified lumped parameters model is considered. As shown in Figure 1 it is assumed that the hosting structure is composed by a modal mass $m_{1}$, a damper $c_{1}$ and a stiffness $k_{1}$ and is excited by a primary force $f_{p}$. The inertial actuator is composed by a block mass $m_{2}$, mounted on a suspension of stiffness $k_{2}$ and damping $c_{2}$ in parallel with a reactive actuator. At frequencies above its fundamental resonance frequency, the inertial actuator produces a sky-hook active force $f_{a}$ proportional to the absolute velocity of the modal mass $m_{1}$ via the control gain $g$.

The equation of motion of the system shown in Figure 1 can be written in a matrix form as:

$$
\mathbf{M} \ddot{\mathbf{x}}(t)+\mathbf{C} \dot{\mathbf{x}}(t)+\mathbf{K x}(t)=\boldsymbol{f}(t)
$$

where $\mathbf{M}$ is the mass matrix, $\mathbf{K}$ is the stiffness matrix and $\mathbf{C}$ is the damping matrix given by:

$$
\mathbf{M}=\left[\begin{array}{cc}
m_{1} & 0 \\
0 & m_{2}
\end{array}\right], \mathbf{K}=\left[\begin{array}{cc}
k_{1}+k_{2} & -k_{2} \\
-k_{2} & k_{2}
\end{array}\right], \mathbf{C}=\left[\begin{array}{cc}
c_{1}+c_{2}-g & -c_{2} \\
-c_{2}+g & c_{2}
\end{array}\right]
$$


$\mathbf{x}(t)=\left[\begin{array}{ll}x_{1}(t) & x_{2}(t)\end{array}\right]^{T}$ is the column vector containing the displacements of the two masses $x_{1}$ and $x_{1}$ and $\mathbf{f}(t)=\left[\begin{array}{lll}f_{\mathrm{p}}(t) & 0\end{array}\right]^{T}$ is the column vector of primary excitation.

Assuming the excitation to be harmonic for the time being and expressing the force and the steady-state response in exponential form, equation (1) becomes:

$$
\mathbf{S}(\mathrm{j} \omega) \mathbf{x}(\mathrm{j} \omega)=\mathbf{f}(\mathrm{j} \omega)
$$

where

$$
\mathbf{S}(\mathrm{j} \omega)=-\omega^{2} \mathbf{M}+\mathrm{j} \omega \mathbf{C}+\mathbf{K}
$$

is the dynamic stiffness matrix. The solution of equation (3) can be obtained as:

$$
\mathbf{x}(\mathrm{j} \omega)=\mathbf{S}^{-1}(\mathrm{j} \omega) \mathbf{f}(\mathrm{j} \omega)
$$

Integrating equation (5) to obtain the velocities yields:

$$
\dot{\mathbf{x}}(\mathrm{j} \omega)=\mathbf{Y}(\mathrm{j} \omega) \mathbf{f}(\mathrm{j} \omega)
$$

where $\dot{\mathbf{x}}(\mathrm{j} \omega)=\mathrm{j} \omega \mathbf{x}(\mathrm{j} \omega)$ and $\mathbf{Y}(\mathrm{j} \omega)=\mathrm{j} \omega \mathbf{S}^{-1}(\mathrm{j} \omega)$ is the mobility matrix. Using the expression of $\mathbf{M}, \mathbf{K}$ and $\mathbf{C}$ of equation (2), the steady state complex response of the system can be expressed in terms of the input and transfer frequency response functions (FRF) as:

$$
\begin{aligned}
& Y_{11}(j \omega)=\frac{\dot{x}_{1}}{f_{\mathrm{p}}}= \\
& =\frac{\mathrm{j}_{2} \omega-\mathrm{c}_{2} \omega^{2}-\mathrm{j} m_{2} \omega^{3}}{k_{1} k_{2}+\mathrm{jc}_{2} k_{1} \omega+\mathrm{jc}_{1} k_{2} \omega-\mathrm{c}_{1} \mathrm{c}_{2} \omega^{2}-k_{2} m_{1} \omega^{2}-k_{1} m_{2} \omega^{2}-k_{2} m_{2} \omega^{2}-\mathrm{jc}_{2} m_{1} \omega^{3}-} \\
& Y_{21}(j \omega)=\frac{\dot{x}_{2}}{f_{\mathrm{p}} m_{2} \omega^{3}-\mathrm{jc}_{2} m_{2} \omega^{3}+g m_{2} \omega^{2}+m_{1} m_{2} \omega^{4}}= \\
& =\frac{\mathrm{j} k_{2} \omega-\mathrm{c}_{2} \omega^{2}+g \omega^{2}}{k_{1} k_{2}+\mathrm{jc}_{2} k_{1} \omega+\mathrm{jc}_{1} k_{2} \omega-\mathrm{c}_{1} \mathrm{c}_{2} \omega^{2}-k_{2} m_{1} \omega^{2}-k_{1} m_{2} \omega^{2}-k_{2} m_{2} \omega^{2}-\mathrm{jc}_{2} m_{1} \omega^{3}-} \\
& \mathrm{jc}_{1} m_{2} \omega^{3}-\mathrm{jc}_{2} m_{2} \omega^{3}+g m_{2} \omega^{2}+m_{1} m_{2} \omega^{4}
\end{aligned}
$$

where $\dot{x}_{1}$ and $\dot{x}_{2}$ are the complex frequency dependent velocities of masses $m_{1}$ and $m_{2}$ respectively. The two FRFs can be expressed in non dimensional form as follows:

$$
\begin{aligned}
& \Gamma=\sqrt{k_{1} m_{1}} \mathrm{Y}_{11}(\mathrm{j} \lambda)=\frac{B_{0}+(\mathrm{j} \lambda) B_{1}+(\mathrm{j} \lambda)^{2} B_{2}+(\mathrm{j} \lambda)^{3} B_{3}}{A_{0}+(\mathrm{j} \lambda) A_{1}+(\mathrm{j} \lambda)^{2} A_{2}+(\mathrm{j} \lambda)^{3} A_{3}+(\mathrm{j} \lambda)^{4} A_{4}} \\
& \Theta=\sqrt{k_{1} m_{1}} \mathrm{Y}_{12}(\mathrm{j} \lambda)=\frac{C_{0}+(\mathrm{j} \lambda) C_{1}+(\mathrm{j} \lambda)^{2} C_{2}+(\mathrm{j} \lambda)^{3} C_{3}}{A_{0}+(\mathrm{j} \lambda) A_{1}+(\mathrm{j} \lambda)^{2} A_{2}+(\mathrm{j} \lambda)^{3} A_{3}+(\mathrm{j} \lambda)^{4} A_{4}}
\end{aligned}
$$

where the coefficients $A_{0-4}, B_{0-3}$ and $C_{0-3}$ are given by: 


$$
\begin{array}{lll}
A_{0}=v^{2} & B_{0}=0 & C_{0}=0 \\
A_{1}=2 \zeta_{2} v+2 \zeta_{1} v^{2} & B_{1}=v^{2} & C_{1}=v^{2} \\
A_{2}=v^{2}+1+\mu v^{2}+4 \zeta_{1} \zeta_{2} v & B_{2}=2 \zeta_{2} v & C_{2}=2 \zeta_{2} v+4 \alpha \zeta_{2} v \\
A_{3}=2 \zeta_{2} v+2 \zeta_{2} \mu \nu+2 \zeta_{1}+4 \alpha v \zeta_{2} \mu & B_{3}=1 & C_{3}=0 \\
A_{4}=1 & &
\end{array}
$$

The six non dimensional parameters in equations (9) and (10) are defined by:

$$
\begin{gathered}
\mu=m_{2} / m_{1} \\
v=\omega_{2} / \omega_{1} \\
\lambda=\omega / \omega_{1} \\
\zeta_{1}=c_{1} /\left(2 m_{1} \omega_{1}\right) \\
\zeta_{2}=c_{2} /\left(2 m_{2} \omega_{2}\right) \\
\alpha=-g /\left(2 c_{2}\right)
\end{gathered}
$$

where $\mu$ is the mass ratio, $v$ is the frequency ratio, $\lambda$ is the normalised driving frequency, $\zeta_{1}$ and $\zeta_{2}$ are the primary and secondary damping ratios respectively and $\alpha$ is the normalised velocity control feedback gain. The natural frequency of the primary system, $\omega_{1}$, and the natural frequency of the inertial actuator, $\omega_{2}$, are defined as:

$$
\begin{aligned}
& \omega_{1}=\sqrt{k_{1} / m_{1}}, \\
& \omega_{2}=\sqrt{k_{2} / m_{2}} .
\end{aligned}
$$

It should be notice that the system is only conditionally stable, therefore the control gain cannot exceed the value that lead to instability the system. The characteristic equation of the control system is given by the denominator of equations (9) and (10). Applying Routh's stability criterion, the stability of the velocity feedback loop can be studied by writing the array of coefficients as shown in Table 1.

The coefficients of the characteristic equation are all positive and so is the coefficient $b_{1}$. To guarantee the stability of the system the numerator of coefficient $b_{2}$ has to be greater than zero and thus the following condition has to be satisfied:

$$
S_{2} \alpha^{2}+S_{1} \alpha+S_{0}>0
$$

where $S_{2}, S_{1}$ and $S_{0}$ are given by:

$$
\begin{gathered}
S_{2}=-4 \mu^{2} v^{3} \zeta_{2}^{2} \\
S_{1}=2 \mu \nu \zeta_{2}\left(\zeta_{2}+v\left(-(1+\mu) v \zeta_{2}+4 v \zeta_{1}^{2} \zeta_{2}+\zeta_{1}\left(-1+(1+\mu) v^{2}+4 \zeta_{2}^{2}\right)\right)\right) \\
S_{0}=\mu v^{3} \zeta_{1}^{2}+\zeta_{1}\left(1-2 v^{2}+(1+\mu)^{2} v^{4}+4 v^{2} \zeta_{1}^{2}\right) \zeta_{2}+v\left(\mu+4\left(1+(1+\mu) v^{2}\right) \zeta_{1}^{2}\right) \zeta_{2}^{2}+4(1+\mu) v^{2} \zeta_{1} \zeta_{2}^{3}
\end{gathered}
$$




\section{DEFINITION OF THE PERFORMANCE CRITERIA}

In this section the minimisation of the kinetic energy of the primary system and the maximisation of the power dissipated by the inertial actuator are considered as tuning strategies of the controller. Therefore, a performance index to be minimised is the integral of the kinetic energy of the primary mass calculated over the frequency-band $\pm \infty$ defined by:

$$
I_{k}=\frac{m_{1} \mathrm{E}\left[\left|\dot{x}_{1}(\mathrm{t})\right|^{2}\right]}{2 S_{f} \omega_{1} / k_{1}}
$$

where $\mathrm{E}\left[\mathrm{]}\right.$ denotes the expectation value. The performance index $I_{k}$ represents the ratio of the kinetic energy of the primary system to the excitation force with a uniform spectrum density $S_{f}(\omega)$. The unit of $S_{f}(\omega)$ is $\mathrm{N}^{2} \mathrm{~s} / \mathrm{rad}$. The constant $S_{f} \omega_{1} / k_{1}$ is introduced to ensure that the performance index is dimensionless. The mean squared value of the velocity of the primary mass can be written as:

$$
\mathrm{E}\left[\left|\dot{x}_{1}(\mathrm{t})\right|^{2}\right]=\frac{S_{f} \omega_{1}}{m_{1} k_{1}} \int_{-\infty}^{+\infty}|\Gamma|^{2} \mathrm{~d} \lambda
$$

Substituting equation (16) in equation (15) yields:

$$
I_{k}=\frac{1}{2} \int_{-\infty}^{+\infty}|\Gamma|^{2} \mathrm{~d} \lambda
$$

Thus, substituting equation (9) in (17) gives:

$$
I_{k}=\frac{1}{2} \int_{-\infty}^{+\infty}\left|\frac{B_{0}+(\mathrm{j} \lambda) B_{1}+(\mathrm{j} \lambda)^{2} B_{2}+(\mathrm{j} \lambda)^{3} B_{3}}{A_{0}+(\mathrm{j} \lambda) A_{1}+(\mathrm{j} \lambda)^{2} A_{2}+(\mathrm{j} \lambda)^{3} A_{3}+(\mathrm{j} \lambda)^{4} A_{4}}\right|^{2} \mathrm{~d} \lambda
$$

Equation (18) can be integrated using the formula in reference [27] leading to:

$$
I_{k}=\frac{1}{2} \frac{G_{1} \alpha+G_{0}}{F_{2} \alpha^{2}+F_{1} \alpha+F_{0}}
$$

where the coefficients $G_{0,1}$ and $F_{0,1,2}$ are given in Table A.1.

The power absorbed by the passive damper $c_{2}$ is equal to:

$$
P_{\text {pas }}(\omega)=\frac{1}{2} \operatorname{Re}\left\{f_{\mathrm{d}}^{*}(\mathrm{j} \omega)\left[\dot{x}_{1}(\mathrm{j} \omega)-\dot{x}_{2}(\mathrm{j} \omega)\right]\right\}
$$

where $*$ denotes complex conjugate and the force $f_{\mathrm{d}}$ is the force produced by the damper given by:

$$
f_{\mathrm{d}}(\mathrm{j} \omega)=c_{2}\left(\dot{x}_{1}(\mathrm{j} \omega)-\dot{x}_{2}(\mathrm{j} \omega)\right)
$$

Substituting equation (21) in (20) the power passively dissipated by the inertial actuator becomes:

$$
P_{\text {pas }}(\omega)=\frac{1}{2} c_{2}\left|\dot{x}_{1}(\mathrm{j} \omega)-\dot{x}_{2}(\mathrm{j} \omega)\right|^{2}
$$

In this case the non-dimensional performance index is defined by: 


$$
I_{p a s}=\frac{c_{2} \mathrm{E}\left[\left|\dot{x}_{1}(t)-\dot{x}_{2}(\mathrm{t})\right|^{2}\right]}{4 \pi S_{f} \omega_{1} / k_{1}}
$$

which represents the ratio of power passively absorbed by the actuator to that generated by excitation force with a spectrum density $S_{f}$ acting on a damper of value $k_{1} /\left(\pi \omega_{1}\right)$. The constant $2 \pi S_{f} \omega_{1} / k_{1}$ is introduced to ensure that the performance index is dimensionless. The mean squared value of the relative velocity times the mechanical damping $c_{2}$ can be expressed as follow:

$$
c_{2} \mathrm{E}\left[\left(\dot{x}_{1}(t)-\dot{x}_{2}(\mathrm{t})\right)^{2}\right]=\frac{S_{f} \omega_{1}^{2}}{k_{1}} 2 \zeta_{2} \mu \nu \int_{-\infty}^{+\infty}|\Gamma-\Theta|^{2} \mathrm{~d} \lambda
$$

Thus the performance index becomes:

$$
I_{\text {pas }}=\frac{\zeta_{2} \mu \nu}{2 \pi} \int_{-\infty}^{+\infty}\left|\frac{D_{0}+(\mathrm{j} \lambda) D_{1}+(\mathrm{j} \lambda)^{2} D_{2}+(\mathrm{j} \lambda)^{3} D_{3}}{A_{0}+(\mathrm{j} \lambda) A_{1}+(\mathrm{j} \lambda)^{2} A_{2}+(\mathrm{j} \lambda)^{3} A_{3}+(\mathrm{j} \lambda)^{4} A_{4}}\right|^{2} \mathrm{~d} \lambda
$$

where

$$
\begin{gathered}
D_{0}=B_{0}-C_{0}=0 \\
D_{1}=B_{1}-C_{1}=0 \\
D_{2}=B_{2}-C_{2}=-4 \alpha \nu \zeta_{2} \\
D_{3}=B_{3}-C_{3}=1
\end{gathered}
$$

The integral over the frequency band between $\pm \infty$ of equation (25) can be calculated using the expression given in reference [27], leading to:

$$
I_{\text {pas }}=\frac{1}{2} \frac{H_{2} \alpha^{2}+H_{1} \alpha+H_{0}}{F_{2} \alpha^{2}+F_{1} \alpha+F_{0}}
$$

where the coefficients $H_{0,1,2}$ are given in Table A.1.

The frequency dependent power absorbed by the active damper is equal to:

$$
P_{a c t}(\omega)=\frac{1}{2} \operatorname{Re}\left\{f_{\mathrm{a}}^{*}(\mathrm{j} \omega)\left[\dot{x}_{1}(\mathrm{j} \omega)-\dot{x}_{2}(\mathrm{j} \omega)\right]\right\}
$$

Assuming the force $f_{a}$ is proportional to the velocity $\dot{x}_{1}(\mathrm{j} \omega)$, equation (28) can be written as:

$$
P_{a c t}(\omega)=-\frac{g}{2} \operatorname{Re}\left\{\dot{x}_{1}^{*}(\mathrm{j} \omega)\left[\dot{x}_{1}(\mathrm{j} \omega)-\dot{x}_{2}(\mathrm{j} \omega)\right]\right\}=-\frac{g}{2}\left[\left|\dot{x}_{1}(t)\right|^{2}-\operatorname{Re}\left\{\dot{x}_{1}^{*}(\mathrm{j} \omega) \dot{x}_{2}(\mathrm{j} \omega)\right\}\right]
$$

After some manipulations equation (29) can written as:

$$
P_{\text {act }}(\omega)=-\frac{g}{4}\left[\left|\dot{x}_{1}(\mathrm{j} \omega)\right|^{2}+\left|\dot{x}_{1}(\mathrm{j} \omega)-\dot{x}_{2}(\mathrm{j} \omega)\right|^{2}-\left|\dot{x}_{2}(\mathrm{j} \omega)\right|^{2}\right]
$$

In this case the non-dimensional performance index is defined by:

$$
I_{a c t}=-\frac{g \mathrm{E}\left[\left|\dot{x}_{1}(\mathrm{t})\right|^{2}+\left|\dot{x}_{1}(t)-\dot{x}_{2}(\mathrm{t})\right|^{2}-\left|\dot{x}_{2}(\mathrm{t})\right|^{2}\right]}{4 \pi S_{f} \omega_{1} / k_{1}}
$$

which represents the ratio of actively absorbed power to that generated by excitation force with a spectrum density $S_{f}$ acting on a damper of value $k_{1} /\left(\pi \omega_{1}\right)$. The same constant, $2 \pi S_{f} \omega_{1} / k_{1}$, is introduced to ensure that the performance index is dimensionless as 
introduced above. The mean squared value of the relative velocity times the control gain $g$ can be expressed as follow:

$$
\begin{aligned}
g \mathrm{E}\left[\left|\dot{x}_{1}(\mathrm{t})\right|^{2}+\left|\dot{x}_{1}(t)-\dot{x}_{2}(\mathrm{t})\right|^{2}-\left|\dot{x}_{2}(\mathrm{t})\right|^{2}\right] & \\
= & \frac{S_{\mathrm{f}} \omega_{1}^{2}}{k_{1}} 2 \zeta_{2} \mu \nu\left[\int_{-\infty}^{+\infty}|\Gamma|^{2} \mathrm{~d} \lambda+\int_{-\infty}^{+\infty}|\Gamma-\Theta|^{2} \mathrm{~d} \lambda-\int_{-\infty}^{+\infty}|\Theta|^{2} \mathrm{~d} \lambda\right]
\end{aligned}
$$

Thus, substituting the expressions of $\Gamma$ and $\Theta$ in equation (32) and calculating the integrals over the frequency band between $\pm \infty$ using the expression given in reference [27] the performance index becomes:

$$
I_{\text {act }}=\frac{L_{2} \alpha^{2}+L_{1} \alpha}{F_{2} \alpha^{2}+F_{1} \alpha+F_{0}}
$$

where the coefficients $L_{1,2}$ are given in Table A.1. The frequency dependent total power absorbed by the inertial actuator system, $P_{\text {tot }}(\omega)$ is equal to that dissipated by the passive damper $c_{2}, P_{\text {pas }}(\omega)$, plus the power actively dissipated by the actuator, $P_{a c t}(\omega)$. Thus the non-dimensional performance index for the total power absorbed is given by the sum of $I_{\text {act }}$ plus $I_{\text {pas }}$ yielding to:

$$
I_{p}=\frac{1}{2} \frac{\left(2 L_{2}+H_{2}\right) \alpha^{2}+\left(2 L_{1}+H_{1}\right) \alpha+H_{0}}{F_{2} \alpha^{2}+F_{1} \alpha+F_{0}}
$$

Although the denominators are the same in equations (19) and (34), the dependence of their numerators on $\zeta_{2}, v$ and $\alpha$ is clearly different.

\section{MINIMISATION OF KINETIC ENERGY AND MAXIMISATION POWER ABSORBED}

In this section the tuning of the damping ratio and natural frequency of the inertial actuator and the feedback control gain is consider to either minimise the kinetic energy of mass $m_{1}$ or maximise the power absorbed by the controller. In order to minimise the kinetic energy of mass $m_{1}$, the following conditions have to be satisfied:

$$
\left\{\begin{array}{l}
\frac{\partial I_{k}}{\partial \zeta_{2}}=0 \\
\frac{\partial I_{k}}{\partial v}=0 \\
\frac{\partial I_{k}}{\partial \alpha}=0
\end{array}\right.
$$

while to maximise the total power absorbed by the inertial actuator the following conditions have to be satisfied: 


$$
\left\{\begin{array}{l}
\frac{\partial I_{p}}{\partial \zeta_{2}}=0 \\
\frac{\partial I_{p}}{\partial v}=0 \\
\frac{\partial I_{p}}{\partial \alpha}=0
\end{array}\right.
$$

Differentiating the performance index $I_{k}$ expressed in equation (19) with respect to $\zeta_{2}, v$ and $\alpha$ and setting these equal to zero, yields a system of three equations:

$$
\left\{\begin{array}{c}
-\mu \nu \Omega=0 \\
-\zeta_{2} \mu \Pi=0 \\
-\mu \nu \zeta_{2} \Psi=0
\end{array}\right.
$$

Following the same procedure, the partial derivates of the performance index $I_{p}$ expressed in equation (36) are given by:

$$
\left\{\begin{array}{c}
\mu \nu \zeta_{1} \Omega=0 \\
\mu \zeta_{1} \zeta_{2} \Pi=0 \\
\mu \nu \zeta_{1} \zeta_{2} \Psi=0
\end{array}\right.
$$

where $\Omega, \Pi$ and $\Psi$ are given in Table A.2.

Equation (37) and (38) are both satisfied for $\zeta_{2}=0$ and $v=0$ although this solution is physically meaningless since it sets to zero the internal damping of the actuator and its mechanical stiffness. The other non trivial solutions can be found setting to zero the terms $\Omega$, $\Pi$ and $\Psi$. Due to the complexity of these expressions no explicit solutions have been found. However, equations (37) and (38) are both satisfied for the same values of $\zeta_{2}, v$ and $\alpha$, which means that if a minimum of the kinetic energy and a maximum of the power absorbed exist, they correspond.

\section{POWER INPUT}

From the energy balance, in steady state conditions, the power input into the system is equal to the power dissipated by damper 1 plus the power dissipated by the controller. The power dissipated by damper 1 is given by:

$$
P_{1}(\omega)=\frac{1}{2} c_{1}\left|\dot{x}_{1}(\mathrm{j} \omega)\right|^{2}
$$

In order to obtain a dimensionless formulation, as done in the previous sections, a dimensionless index relative to $P_{1}(\omega)$ can be defined by:

$$
I_{p 1}=\frac{c_{1} E\left[\left|\dot{x}_{1}(t)\right|^{2}\right]}{4 \pi S_{f} \omega_{1}^{2} / k_{1}}
$$

Therefore the sum of $I_{p 1}$ and $I_{p}$ gives the dimensionless power input into the system. The mean squared value of the velocity of mass $m_{1}$ times the mechanical damping $c_{1}$ can be expressed as follow: 


$$
c_{1} E\left[\left|\dot{x}_{1}(t)\right|^{2}\right]=\frac{S_{\mathrm{f}} \omega_{1}^{2}}{c_{1}} 2 \zeta_{1} \int_{-\infty}^{+\infty}|\Gamma|^{2} d \lambda
$$

Substituting equation (41) in (40), the performance index becomes:

$$
I_{p 1}=\frac{\zeta_{1}}{2 \pi} \int_{-\infty}^{+\infty}\left|\frac{B_{0}+(j \lambda) B_{1}+(j \lambda)^{2} B_{2}+(j \lambda)^{3} B_{3}}{A_{0}+(j \lambda) A_{1}+(j \lambda)^{2} A_{2}+(j \lambda)^{3} A_{3}+(j \lambda)^{4} A_{4}}\right|^{2} d \lambda=\frac{\zeta_{1}}{\pi} I_{k}
$$

The power input into the system can be calculated as the sum of equation (27) and (42). Summing the two expressions the result is $1 / 4$, which, multiplied by the constant $2 \pi S_{\mathrm{f}} \omega_{1}^{2} / k_{1}$ used to make the performance indices $I_{p 1}$ and $I_{p}$ dimensionless leads to:

$$
P_{\text {in }}=\frac{\pi}{2 m_{1}}
$$

where $P_{\text {in }}$ is the power input into the system. Equation (43) indicates that, when excited by a random force with a flat spectrum, the power input into the system only depends on the mass $m_{1}$. This means that the derivates of $I_{p 1}$ are equal to the derivates of $I_{p}$. Because the kinetic energy of the primary system is proportional to the power dissipated by the damper 1 its derivates are proportional to the derivates of $I_{p}$ and therefore the minimisation of the kinetic energy corresponds to the maximisation of the power absorbed by the controller as shown in the previous section.

\section{TUNING OF THE CONTROL GAIN}

In order to implement skyhook damping with a velocity feedback loop using an inertial actuator, the natural frequency of the actuator should be lower than the first natural frequency of the primary system and the resonance of the actuator has to be well damped to improve control stability $[17,18,28]$. However a low resonance is difficult to achieve in practice because a soft suspension will cause large static deflection and will increase the risk that the inertial mass could hit the ends-stop, potentially leading to instability [29]. Thus the physical properties of the inertial actuator represented by the dimensionless parameters, $\mu, v$ and $\zeta_{2}$ are usually selected taking into account these practical limits. The control gain, represented by the dimensionless parameter $\alpha$, could be tuned online to optimise the control performance, that is by minimising the kinetic energy of the primary system or equivalently maximising the power absorbed by the actuator. Research on this topic has been carried and an algorithm that automatically tune the control gain of decentralised control units has been proposed to maximise their power absorbed making the controller self-tuneable [25, 26].

As shown in equation (37) and (38), setting to zero the derivative of $I_{k}$ and $I_{p}$ with respect of $\alpha$, the quadratic equation $\Psi=0$ in the unknown $\alpha$ is obtained. The solution of the latter equation allows the analytical expression of $\alpha_{o p t}$ to be found and the solution of inequality 
(13) gives an analytical expression for the maximum stable gain $\alpha_{\max }$ which are not reported here for brevity. Due to the complexity of these expressions it has not been possible to guarantee that the solutions are real in the general case. However, to gain a better understanding of the physics behaviour of the system, simulation results are presented using the parameters summarised in Table 2 .

Figure 2 shows the power spectral density of the dimensionless velocity of the primary mass for $\alpha=0$ (solid line), $\alpha=\alpha_{o p t}$ (dashed line) and $\alpha=\alpha_{\text {max }}$ (dotted line). When no active control is implemented the spectrum is dominated by the resonance of the primary system. At about $\lambda=v$ the spectrum presents a small peak due to the resonance of the inertial actuator. When the control gain is increased the response of the primary system is reduced at resonance but it is also amplified at the resonance frequency of the inertial actuator. When the control gain approaches the stability limit the response of the system at the resonance frequency of the inertial actuator will tend to infinity as shown by the dotted line in Figure 2. Figure 3 shows the dimensionless kinetic energy of the primary system $I_{k}$ (a) and the dimensionless power absorbed by the inertial actuator $I_{p}$ (b). The graphs show that the kinetic energy decreases as the control gain is increased, reaching a minimum (red circle) before it starts to increases again when the control gain approaches the stability limit. The power absorbed increases when the control gain increases, it reaches a maximum (red circle) and it drops when the control gain approaches the stability limit. As demonstrated in the previous sections the minimum of the kinetic energy corresponds to the maximum of the power absorbed.

Figure 4(a) and 4(b) show the maximum stable gain $\alpha_{\max }$ and the reduction in the kinetic energy $I_{k}$ as function of $\zeta_{2}$ and $v$ respectively, as predicted by the theory. The plots show that for small values of $v, \alpha_{\max }$ gets larger and better reductions of the kinetic energy are obtained. In both plots the variation of $\zeta_{2}$ has marginal effect on the values of $\alpha_{\max }$ and $I_{k}$. This confirms that the resonance of the inertial actuator has to be as low as possible compared to the resonance of the primary system to improve control stability. In the extreme case of $v$ equal to zero, the inertial mass of the actuator is grounded and the active force is generated reacting of a rigid structure, therefore the system is unconditionally stable $\left(\alpha_{\max } \rightarrow+\infty\right)$.

Figure 5 shows the ratio between the optimal control gain $\alpha_{o p t}$ and maximum stable gain $\alpha_{\max }$ as function of the $\zeta_{2}$ and $v$. The plot shows that the optimal gain approaches the maximum stable gain for low values of $v$ and diverges from the maximum stable gain for values of $v$ near 1 . 


\section{CONCLUSIONS}

This paper has presented a theoretical study on the implementation of a velocity feedback control loop in order to reduce the response of a structure excited by a broadband white noise disturbance. The controller consists of an inertial actuator driven with a signal proportional to the velocity measured by an ideal sensor at the actuator footprint.

In the first part of this study it is demonstrated that for a damped host structure, modelled as a single degree of freedom, the ratios of natural frequencies, the actuator mechanical damping and the control gain that maximise the power absorbed by the controller are the same as those that minimise the kinetic energy of the host structure. In fact it has been demonstrated that the simultaneous equations of the partial derivatives of the kinetic energy and the power absorbed are satisfied for the same values of the ratios of natural frequencies, the actuator mechanical damping and the control gain although their analytical expressions could not be found due to the complexity of these expressions. Therefore, the effect of maximising the absorbed power is equivalent to minimising the kinetic energy of the host structure.

In the second part only the feedback control gain has been considered in the tuning process. In practical cases of active vibration control, the mechanical damping of the actuator and its natural frequency are chosen as a compromise of stability and performance. It is demonstrated that the maximum stable gain and optimal gain can be obtained analytically as solutions of two quadratic equations.

This is an important finding that may provide a method of self-tuning such a controller. If the power dissipated by the controller could be measured and the disturbance was stationary, a tuning strategy might be used that is similar to that used for feedback controllers in references [25-26].

\section{APPENDIX A}

Table A.1: Coefficients of equations (19), (27) and (33)

\begin{tabular}{|l|}
\hline$F_{0}=\left(4 v^{2} \zeta_{1}^{3} \zeta_{2}+\mu \nu \zeta_{2}^{2}+\zeta_{1} \zeta_{2}\left(1-2 v^{2}+(1+\mu)^{2} v^{4}+4(1+\mu) v^{2} \zeta_{2}^{2}\right)+\zeta_{1}^{2}\left(\mu v^{3}+4\left(v+(1+\mu) v^{3}\right) \zeta_{2}^{2}\right)\right)$ \\
\hline$F_{1}=2 \mu \nu \zeta_{2}\left(-\left(-1+(1+\mu) v^{2}\right) \zeta_{2}+4 v^{2} \zeta_{1}^{2} \zeta_{2}+v \zeta_{1}\left(-1+(1+\mu) v^{2}+4 \zeta_{2}^{2}\right)\right)$ \\
\hline$F_{2}-4 \mu^{2} v^{3} \zeta_{2}^{2}$ \\
\hline$G_{0}=4 v^{2} \zeta_{1}^{2} \zeta_{2}+\zeta_{2}\left(1-2 v^{2}+(1+\mu) v^{4}+4 v^{2} \zeta_{2}^{2}\right)+\zeta_{1}\left(\mu v^{3}+4\left(v+v^{3}\right) \zeta_{2}^{2}\right)$ \\
\hline$G_{1}=2 \mu v^{2}\left(-1+v^{2}\right) \zeta_{2}$ \\
\hline$H_{0}=\mu \nu \zeta_{2}\left(\zeta_{2}+4 v^{2} \zeta_{1}^{2} \zeta_{2}+\zeta_{1}\left((1+\mu) v^{3}+4 v \zeta_{2}^{2}\right)\right)$ \\
\hline$H_{1}=-2 \mu^{2} v^{3} \zeta_{2}^{2}$ \\
\hline$H_{2}=16 \mu v^{3} \zeta_{2}^{3}\left(\nu \zeta_{1}+\zeta_{2}\right)$ \\
\hline$L_{1}=\mu \nu \zeta_{2}\left(-\left(-1+v^{2}\right) \zeta_{2}+4 v^{2} \zeta_{1}^{2} \zeta_{2}+\zeta_{1}\left(\mu v^{3}+4 v \zeta_{2}^{2}\right)\right)$ \\
\hline
\end{tabular}


Table A.2: coefficients of equations (37) and (38)

\begin{tabular}{|l}
$\Omega=\left(-16(1+2 \alpha) v^{4} \zeta_{1}^{4} \zeta_{2}^{2}-8(1+2 \alpha) v^{3} \zeta_{1}^{3} \zeta_{2}\left(\mu v^{2}+4 \zeta_{2}^{2}\right)+\left(-1-2 \alpha+2 \alpha(1+\mu+2 \alpha \mu) v^{2}\right) \zeta_{2}^{2}(1-2(1\right.$ \\
$\left.+\alpha \mu) v^{2}+(1+\mu+2 \alpha \mu) v^{4}-4 v^{2} \zeta_{2}^{2}\right)+2 v \zeta_{1} \zeta_{2}\left(\mu v^{2}\left(-1-2 \alpha+2 \alpha(1+\mu+2 \alpha \mu) v^{2}\right)\right.$ \\
$\left.-4\left(1-2 v^{2}+2 \alpha\left(-1+v^{2}\right)\left(-1+(1+\mu+2 \alpha \mu) v^{2}\right)\right) \zeta_{2}^{2}\right)+v^{2} \zeta_{1}^{2}\left(-\mu(1+\mu+2 \alpha \mu) v^{4}\right.$ \\
$\left.\left.-4 \zeta_{2}^{2}\left(2+4 \alpha-(1+2 \alpha)(3-2 \mu+4 \alpha \mu) v^{2}+2 \alpha(1+\mu+2 \alpha \mu) v^{4}+(4+8 \alpha) \zeta_{2}^{2}\right)\right)\right)$ \\
\hline$\Pi=\left(-\mu(1+\mu+2 \alpha \mu) v^{6} \zeta_{1}^{2}-2 v^{3} \zeta_{1}\left(2+\mu+2 \alpha \mu-2(1+\alpha \mu)(1+\mu+2 \alpha \mu) v^{2}+4(1+\mu+2 \alpha \mu) v^{2} \zeta_{1}^{2}\right) \zeta_{2}\right.$ \\
$-\left(1+2 \alpha-2(-1+\alpha+2 \alpha(1+2 \alpha) \mu) v^{2}+(1+\mu+2 \alpha \mu)\left(-3-2 \alpha+4 \alpha^{2} \mu\right) v^{4}\right.$ \\
$+2 \alpha(1+\mu+2 \alpha \mu)^{2} v^{6}+4 v^{2} \zeta_{1}^{2}\left(2+4 \alpha+(1+2 \mu-2 \alpha(3+4 \alpha \mu)) v^{2}-2 \alpha(1+\mu\right.$ \\
$\left.\left.+2 \alpha \mu) v^{4}+4(1+2 \alpha) v^{2} \zeta_{1}^{2}\right)\right) \zeta_{2}^{2}+8 v \zeta_{1}\left(-1+v^{2}+2 \alpha\left(-1+v^{2}\left(2+v^{2}+(1+2 \alpha) \mu(1\right.\right.\right.$ \\
$\left.\left.\left.\left.\left.+v^{2}\right)\right)\right)-4(1+2 \alpha) v^{2} \zeta_{1}^{2}\right) \zeta_{2}^{3}+4 v^{2}\left(1+2 \alpha+2 \alpha(1+\mu+2 \alpha \mu) v^{2}-4(1+2 \alpha) \zeta_{1}^{2}\right) \zeta_{2}^{4}\right)$ \\
\\
$+2 \mu \nu^{3} \zeta_{1}\left(-1+(1+2 \alpha \mu) v^{2}-4 v^{2} \zeta_{1}^{2}\right) \zeta_{2}-\left(-1+(3+4 \alpha \mu) v^{2}-(3+2 \mu(1+2 \alpha(2\right.$ \\
$\left.+\alpha \mu)) v^{4}+(1+\mu+2 \alpha \mu)^{2} v^{6}-4 v^{2} \zeta_{1}^{2}\left(2+v^{2}\left(-3+v^{2}+\mu\left(2-4 \alpha+v^{2}\right)\right)+4 v^{2} \zeta_{1}^{2}\right)\right) \zeta_{2}^{2}$ \\
$-8 v \zeta_{1}\left(-1+v^{2}\left(1+2 \alpha \mu\left(1+v^{2}\right)\right)-2 v^{2}\left(2+v^{2}\right) \zeta_{1}^{2}\right) \zeta_{2}^{3}-4 v^{2}\left(-1+(1+\mu+4 \alpha \mu) v^{2}\right.$ \\
$\left.\left.-4\left(1+2 v^{2}\right) \zeta_{1}^{2}\right) \zeta_{2}^{4}+16 v^{3} \zeta_{1} \zeta_{2}^{5}\right)$
\end{tabular}

\section{REFERENCES}

[1] H. Frahm, Device for damping vibrations of bodies, US Patent (1911)

[2] J.P. Den Hartog, Mechanical Vibrations, 4th ed., McGraw-Hill, New York, 1956.

[3] T. Asami, O. Nishihara, A.M. Baz, Analytical solutions to $\mathrm{H} \infty$ and $\mathrm{H} 2$ optimization of dynamic vibration absorbers attached to damped linear systems, Journal of vibration and acoustics 124 (2002) 284-295.

[4] S.H. Crandall, W.D. Mark, Random vibration in mechanical systems, Academic Press, 1963.

[5] Y. Iwata, On the construction of the dynamic vibration absorber, Japanese Society of Mechanical Engineering, 820 (1982) 150-152.

[6] S. Krenk, Frequency Analysis of the Tuned Mass Damper, Journal of Applied Mechanics, 72 (2005) 936-942.

[7] S. Krenk, J. Høgsberg, Tuned mass absorbers on damped structures under random load, Probabilistic Engineering Mechanics, 23 (2008) 408-415.

[8] O. Nishihara, T. Asami, Closed-Form Solutions to the Exact Optimizations of Dynamic Vibration Absorbers (Minimizations of the Maximum Amplitude Magnification Factors), Journal of Vibration and Acoustics, 124 (2002) 576-582.

[9] O. Nishihara, H. Matsuhisa, Design of a Dynamic Vibration Absorber for Minimisation of Maximum Amplitude Magnification Factor (Derivation of Algebric Exact Solution), Transactions of the Japan Society of Mechanical Engineers, Ser. C, 62-614 (1997) 3438-3445. 
[10] G.B. Warburton, Optimum absorber parameters for various combinations of response and excitation parameters, Journal of earthquake engineering and structural dynamics 10 (1982) 381-401.

[11] H. Yamaguchi, Damping of Transient vibration by a Dynamic Absorber, Transactions of the Japan Society of Mechanical Engineers, Ser. C, 54 (1988) 561-568.

[12] M. Zilletti, S.J. Elliott, E. Rustighi, Optimisation of dynamic vibration absorbers to minimise kinetic energy and maximise internal power dissipation, Journal of Sound and Vibration, 331 (2012) 4093-4100.

[13] S.J. Elliott, J. Rohlfing, P. Gardonio, Multifunctional design of inertially-actuated velocity feedback controllers, The Journal of the Acoustical Society of America, 131 (2012) $1150-1157$.

[14] A. Preumont, Vibration Control of Active Structures, Kluwer Academic, London, 2002.

[15] S.J. Elliott, Global vibration control through local feedback, in: D. Wagg, I. Bond, P. Weaver, M. Friswell (Eds.) Adaptive Structure: Engineering Applications, Johon Wiley \& Sons Ltd, Chichester, 2007, pp. 59-87.

[16] S.J. Elliott, P. Gardonio, T.C. Sors, M.J. Brennan, Active vibroacoustic control with multiple local feedback loops, The Journal of the Acoustical Society of America, 111 (2002) 908-915.

[17] C. González Díaz, P. Gardonio, Feedback control laws for proof-mass electrodynamic actuators, Smart Materials and Structures, 16 (2007) 1766.

[18] C. González Díaz, C. Paulitsch, P. Gardonio, Active damping control unit using a small scale proof mass electrodynamic actuator, The Journal of the Acoustical Society of America, 124 (2008) 886-897.

[19] J. Rohlfing, S.J. Elliott, P. Gardonio, Feedback compensator for control units with proofmass electrodynamic actuators, Journal of Sound and Vibration, 331 (2012) 3437-3450.

[20] Y.L. Cheung, W.O. Wong, L. Cheng, Minimization of the mean square velocity response of dynamic structures using an active-passive dynamic vibration absorber, The Journal of the Acoustical Society of America, 132 (2012) 197-207.

[21] N. Hirami, An active maximum power absorber for the reduction of noise and vibration, Journal of Sound and Vibration, 200 (1997) 261-279.

[22] N. Hirami, Optimal energy absorption as an active noise and vibration control strategy, Journal of Sound and Vibration, 200 (1997) 243-259.

[23] W. Redman-White, P.A. Nelson, A.R.D. Curtis, Experiments on the active control of flexural wave power flow, Journal of Sound and Vibration, 112 (1987) 187-191.

[24] S.J. Sharp, P.A. Nelson, G.H. Koopmann, A theoretical investigation of optimal power absorptionas a noise control technique, Journal of Sound and Vibration, 251 (2002) 927-935.

[25] M. Zilletti, S.J. Elliott, P. Gardonio, Self-tuning control systems of decentralised velocity feedback, Journal of Sound and Vibration, 329 (2010) 2738-2750.

[26] M. Zilletti, S.J. Elliott, P. Gardonio, E. Rustighi, Experimental implementation of a selftuning control system for decentralised velocity feedback, Journal of Sound and Vibration, 331 (2012) 1-14.

[27] D.E. Newland, An Introduction to Random Vibrations, Spectral \& Wavelet Analysis, Third Edition ed., Dover Publications, Inc, Mineola, New York, 1975.

[28] S.J. Elliott, M. Serrand, P. Gardonio, Feedback Stability Limits for Active Isolation Systems with Reactive and Inertial Actuators, Journal of Vibration and Acoustics, 123 (2001) 250-261.

[29] O.N. Baumann, S.J. Elliott, Destabilization of velocity feedback controllers with stroke limited inertial actuators, The Journal of the Acoustical Society of America, 121 (2007) EL211-EL217. 


\section{FIGURES}

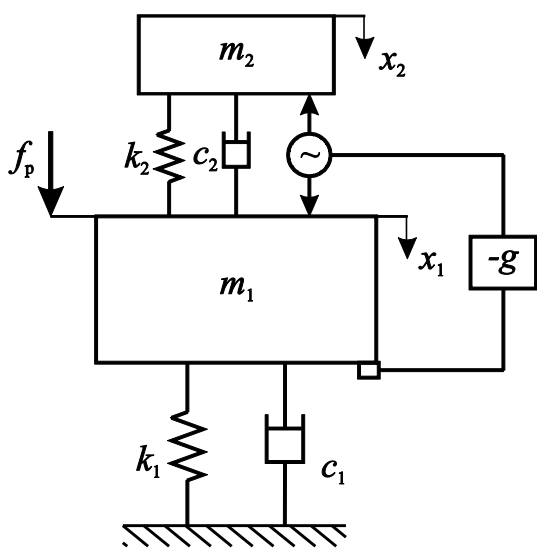

Figure 1: Scheme of the SDOF system with a velocity feedback loop using an inertial actuator

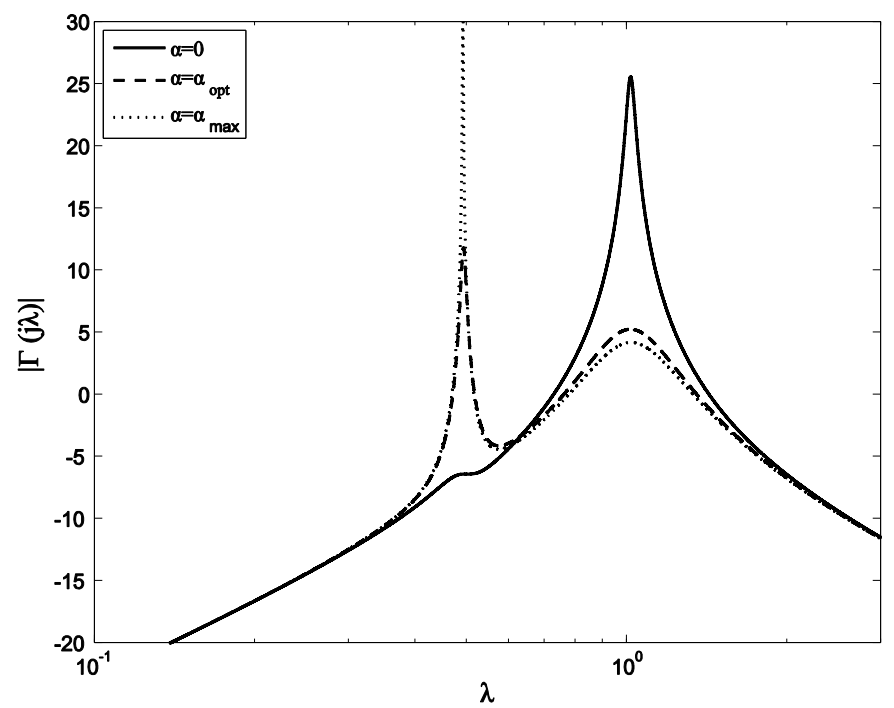

Figure 2: power spectral density of the dimensionless velocity of the primary system for $\alpha=0, \alpha=\alpha_{o p t}$ and $\alpha=\alpha_{\max }$
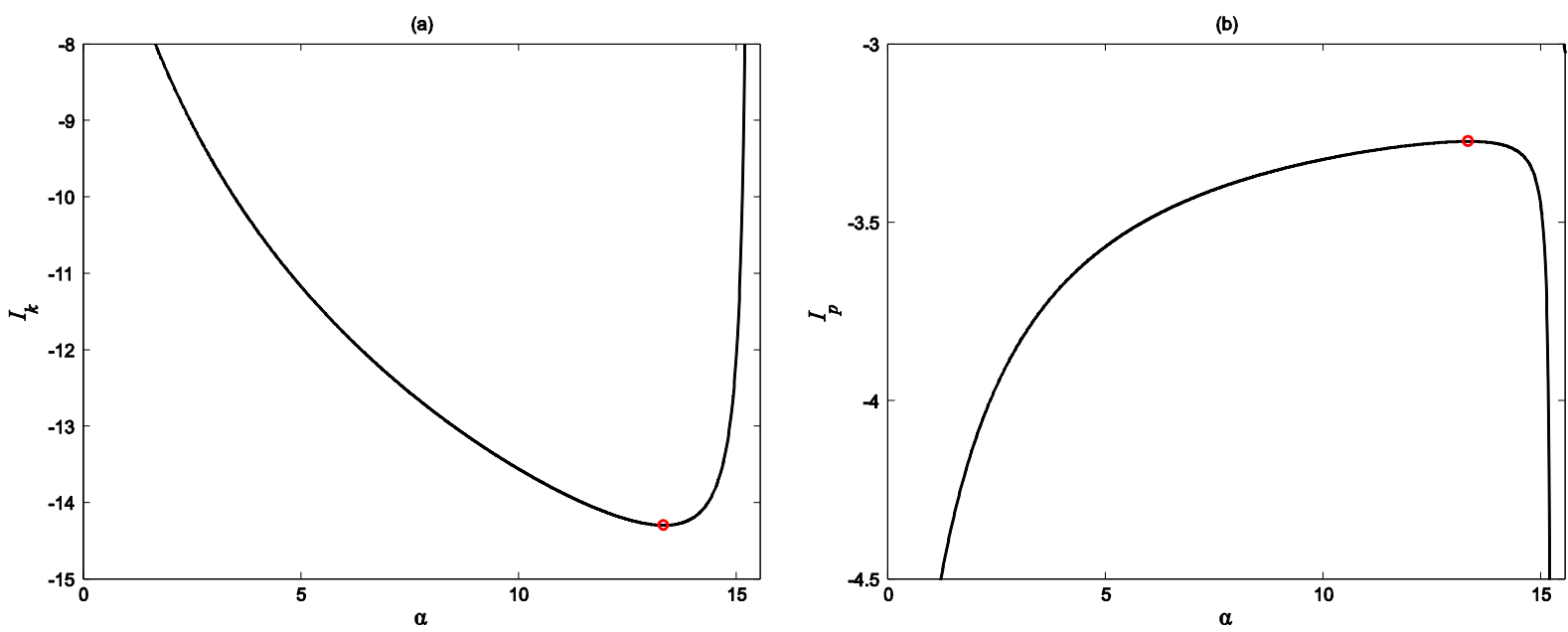

Figure 3: (a) non-dimensional kinetic energy $I_{k}$ (b) non-dimensional power absorbed $I_{p}$ by the inertial actuator as function of $\alpha$. The circular markers (o) indicate the minimum of $I_{k}$ and maximum of $I_{p}$ 

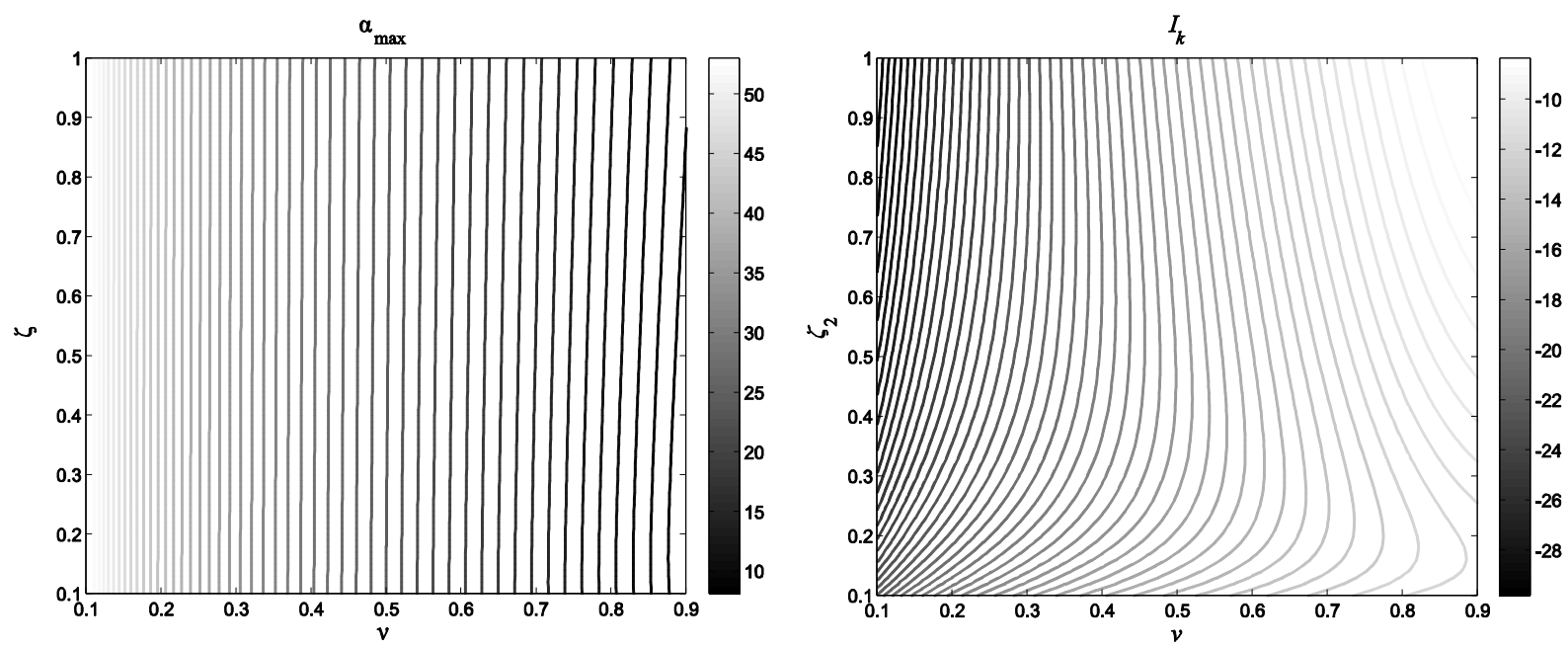

Figure 4: the maximum stable gain $\alpha_{\max }$ in $\mathrm{dB}$ (left plot) and the optimal value of the non-dimensional kinetic energy $I_{k}$ (right plot) as function of $\zeta_{2}$ and $v$

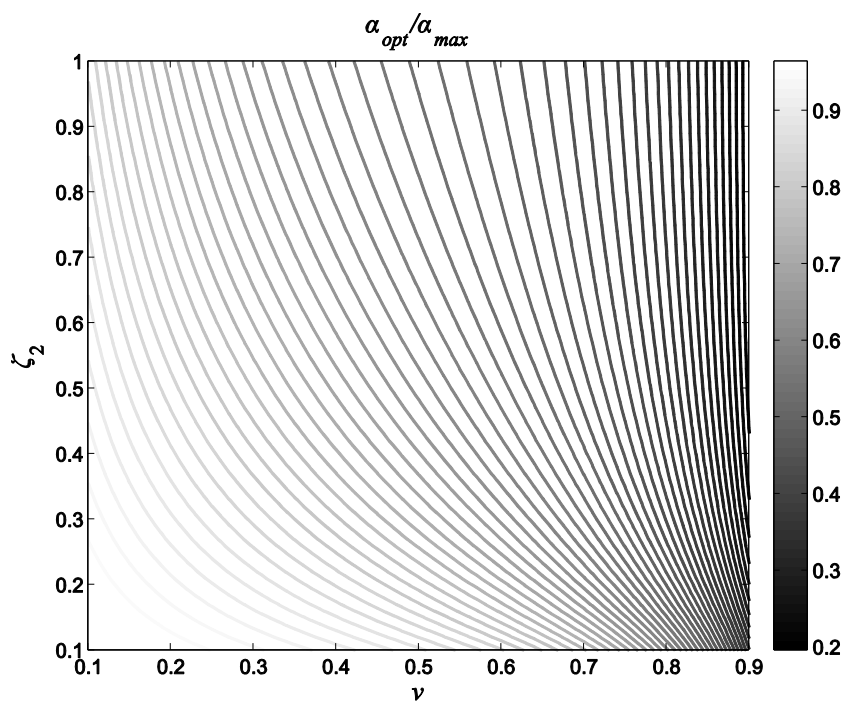

Figure 5: ratio between the optimal gain $\alpha_{o p t}$ and the maximum stable gain $\alpha_{\max }$ as function of $\zeta_{2}$ and $v$

\section{TABLES}

Table 1: Routh's coefficients chart

\begin{tabular}{|c|c|c|}
\hline$A_{4}$ & $A_{2}$ & $A_{0}$ \\
\hline$A_{3}$ & $A_{1}$ & \\
\cline { 1 - 2 }$b_{1}=\frac{A_{3} A_{2}-A_{4} A_{1}}{A_{3}}$ & $A_{0}$ & \\
\cline { 1 - 2 }$b_{2}=\frac{b_{1} A_{1}-A_{3} A_{0}}{b_{1}}$ & \multicolumn{2}{|}{} \\
\cline { 1 - 2 }$A_{0}$ & & \\
\end{tabular}


Table 2: non-dimensional parameters used in the simulations

\begin{tabular}{l|c}
\hline \hline Parameter & Value \\
\hline \hline Mass ratio $\mu$ & 0.1 \\
\hline Natural frequency ratio $v$ & 0.5 \\
\hline Damping ratio of the primary system $\zeta_{1}$ & 0.01 \\
\hline Damping ratio of the inertial actuator $\zeta_{2}$ & 0.1 \\
\hline \hline
\end{tabular}

